\title{
Inflammatory Monocytes Infiltrate the Spiral Ligament and Migrate to the Basilar Membrane After Noise Exposure
}

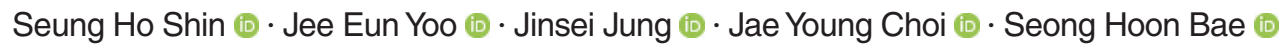 \\ Department of Otorhinolaryngology, Yonsei University College of Medicine, Seoul, Korea
}

Objectives. Noise exposure leads to an increase in the macrophage population. This increment is thought to be caused by the transformation of infiltrated monocytes into macrophages rather than by proliferation of the cochlear resident macrophages. However, studies on infiltrated monocytes in the cochlea are scarce. Thus, we aimed to investigate the infiltration of monocytes and their transformation into macrophages after noise exposure.

Methods. In wild-type and CX3CR1//GPP C57/B6 mice, inflammatory monocytes were identified by immunofluorescence of mouse cochlear cells. The findings were confirmed and quantitated by flow cytometry.

Results. One day after noise exposure, monocytes were identified in the spiral ligament. Flow cytometric analysis confirmed that the monocyte population peaked on post-noise exposure day 1 and decreased thereafter. On day 3 after noise exposure, amoeboid-type macrophages increased in the crista basilaris, and on day 5 , they spread to the basilar membrane.

Conclusion. Infiltrated monocytes were successfully observed 1 day after noise exposure, preceding the increase in the macrophage population. This finding supports the proposal that infiltrated monocytes transform into macrophages.

Keywords. Cochlea; Macrophages; Monocytes; Noise

\section{INTRODUCTION}

Acoustic trauma results in the destruction of hair cells in the organ of Corti and nonsensory cells such as fibrocytes in the spiral limbus and spiral ligament [1]. However, the cellular and molecular mechanisms of acoustic trauma have not been fully elucidated. Recently, inflammation and the immune response have been recognized as important pathophysiological mechanisms involved in acoustic trauma [2-4]. Macrophages, the major innate immune cells, play a critical role in local cochlear immunity

\footnotetext{
- Received May 7, 2021

Revised September 3, 2021

Accepted September 6, 2021

- Corresponding author: Seong Hoon Bae

Department of Otorhinolaryngology, Yonsei University College of Medicine, Severance Hospital, Yonsei University Health System, 50 Yonsei-ro, Seodaemun-gu, Seoul 03722, Korea

Tel: +82-2-2228-3484, Fax: +82-2-393-0580

E-mail: bshsap@naver.com
}

by contributing to inflammation and tissue repair [5]. Resident macrophages are present in the cochlea even before noise damage occurs, and they express CD45, F4/80, ionized calcium-binding adapter molecule 1, CD11b, and Fractalkine receptor (CX3CR1) $[2,4,6,7]$. These macrophages engulf cellular debris, produce proinflammatory cytokines, and present antigens $[5,8]$. Growing evidence suggests that infiltrating monocytes/macrophages are critical for acute inflammation and wound healing in other organs [9-12].

Intense acoustic overstimulation leads to a significant increase of immune cells in the cochlea. Several groups have reported that macrophage levels peak 3-7 days after acoustic trauma $[6,13,14]$. This increased population of macrophages is believed to result from the transformation of infiltrated monocytes, and not from the proliferation of resident macrophages $[2,6]$. However, there is a lack of research on infiltrated monocytes in the cochlea after noise exposure. In this study, we aimed to investigate the infiltration of monocytes and their transformation into

Copyright $@ 2022$ by Korean Society of Otorhinolaryngology-Head and Neck Surgery.

This is an open-access article distributed under the terms of the Creative Commons Attribution Non-Commercial License (https://creativecommons.org/licenses/by-nc/4.0)

which permits unrestricted non-commercial use, distribution, and reproduction in any medium, provided the original work is properly cited. 
macrophages using quantitative flow cytometry. CX3CR1-GFP reporter transgenic mice and the Ly6C antibody were used to visualize the macrophage lineage and inflammatory monocytes, respectively.

\section{MATERIALS AND METHODS}

\section{Animals}

Wild-type C57BL/6 mice and Cx3cr $1^{\mathrm{GFP} / \mathrm{GPP}}$ transgenic C57BL/6 mice were purchased from Jackson Laboratory (Maine) via Orient Bio (Seongnam, Korea). $\mathrm{C} x 3 \mathrm{cr} 1^{+/ / \text {FP }}$ mice were used to visualize the resident macrophages. The mice were housed and maintained according to the animal research requirements, and all procedures were approved by the Institutional Animal Care and Use Committee (approval No. 2019-0182). Mice aged 4-12 weeks of both sexes were used. They were fed ad libitum and housed in cages in an environmentally controlled room under a 12-hour light cycle.

\section{Noise generation}

White noise $(300-10,000 \mathrm{~Hz})$ was generated using a personal computer and an amplifier (R-399; Inter M, Seoul, Korea) and delivered through speakers (290-8L; Altec Lansing, Oklahoma City, OK, USA) in a noise booth. Mice were continuously exposed to $120 \mathrm{~dB}$ peak equivalent sound pressure level (SPL) for 1 hour to induce permanent threshold shift and noise-induced cochlear inflammation.

\section{Audiologic evaluation}

The auditory brainstem response (ABR) was measured after anesthetizing the mice with xylazine $(20 \mathrm{mg} / \mathrm{kg}$ intraperitoneal injection [IP]) and ketamine hydrochloride (30 mg/kg IP). The hearing level of each mouse was checked by measuring the ABR threshold with a Tucker-Davis Technologies (TDT) auditory evoked potential workstation (TDT, Alachua, FL, USA). Both ears of each mouse were stimulated with an ear probe sealed in the ear canal.The ABRs to click and tone stimuli were recorded, and thresholds were obtained for each ear. The ABRs were measured before and at 1 and 14 days after the noise exposure.

\section{H I G H L I G G H T S}

- Noise exposure induced hair cell degeneration and immune cell recruitment.

- Monocytes infiltrated the spiral ligament 1 day after noise exposure.

- Macrophages in the crista basilaris increased in number on post-noise exposure day 3.

- Macrophages spread to the basilar membrane 5 days after noise exposure.

\section{Histological preparation and immunostaining}

After sacrificing the mice in a $\mathrm{CO}_{2}$ chamber, the bilateral temporal bones were dissected and fixed in $4 \%$ paraformaldehyde for 24 hours at $4^{\circ} \mathrm{C}$ after local perfusion with a fixative through the oval and round windows. After fixation, the samples were incubated in 1:3 EDTA (ethylenediaminetetraacetic acid) solution for 24 hours at $4^{\circ} \mathrm{C}$ for decalcification.

For obtaining a whole mount of the organ of Corti sample, decalcified cochleae were cut in half through the apex-oval window axis. Under optical microscopy, the cochleae's lateral wall and bony capsule were carefully removed using forceps and microscissors. Other structures were trimmed while preserving the organ of Corti. All cochlear tissues were separated into apical, middle, and basal turns.

The tissues were blocked with $10 \%$ donkey serum and incubated with dye-conjugated Ly6C antibody (HK1.4, 128016; Biolegend, San Diego, CA, USA) and/or dye-conjugated F4/80 antibody (BM8, 123110, Biolegend) at $4^{\circ} \mathrm{C}$ overnight. The samples were then mounted with a mounting solution (Sigma-Aldrich, St. Louis, MO, USA) and viewed under an LSM780 confocal microscope (Zeiss, Jena, Germany). All immunostaining experiments were repeated in four biological replicates per time point.

\section{Hair cell counts}

After histological preparation, the decalcified cochlea was immunostained with fluorescent- tagged antibodies. FITC-conjugated phalloidin (1:200; P5282; Sigma-Aldrich) was used to stain cochlear hair cells and DAPI (1:5,000; Invitrogen, Carlsbad, CA, USA) for nuclear staining. Basal turns of the cochlea were examined under a confocal microscope (LSM700; Zeiss).

\section{$3 \mathrm{D}$ reconstruction imaging}

Microscopy setting and clearing protocol was performed, as in our previous study [15].Two-photon microscopy (LSM7MP; Carl Zeiss, Oberkochen, Germany) was used for generating imaging data. Zen software (Carl Zeiss) was used for image acquisition and basic image analysis. To create three-dimensional images and videos, IMARIS software (Bitplane, Zurich, Switzerland) was used. Sagittal plane images of $100 \mu \mathrm{m}$ distance were stacked for the sagittal reconstructed image. Basal turn was carefully divided from decalcified cochlea, and mounted on a silicone bond in the petri dish, with basilar membrane positioned at the top. Distilled water was filled between the sample and water-immerse lens of the two-photon microcopy.

\section{Flow cytometry}

The cochleae were obtained from mice after cardiac perfusion with phosphate-buffered saline (PBS). Soft tissue was carefully removed from the cochlea in ice-cold PBS. After moving the cochlea to a new dish filled with ice-cold PBS, the bony capsule was partially removed with special care not to open the bone marrow. After the cochlea's inner tissues were extracted through 
the opening, the remnants were discarded. The cochlear tissue was collected and trypsinized for 10 minutes at $37^{\circ} \mathrm{C}$. The trypsinized tissue was ground and passed through a $40-\mu \mathrm{m}$ filter. The dissociated cells were stained with anti-CD11b (M1/70, 101206, Biolegend), anti-Ly6G (1A8, 127608, Biolegend), and anti-Ly6C (HK1.4, 128016, Biolegend) antibodies (1:200) for 30 minutes. The stained samples were analyzed with a FACSverse II BD flow cytometer (BD Biosciences, Sparks, MD, USA). Each sample consisted of cells from two cochleae from a mouse, and five samples were included in each group.
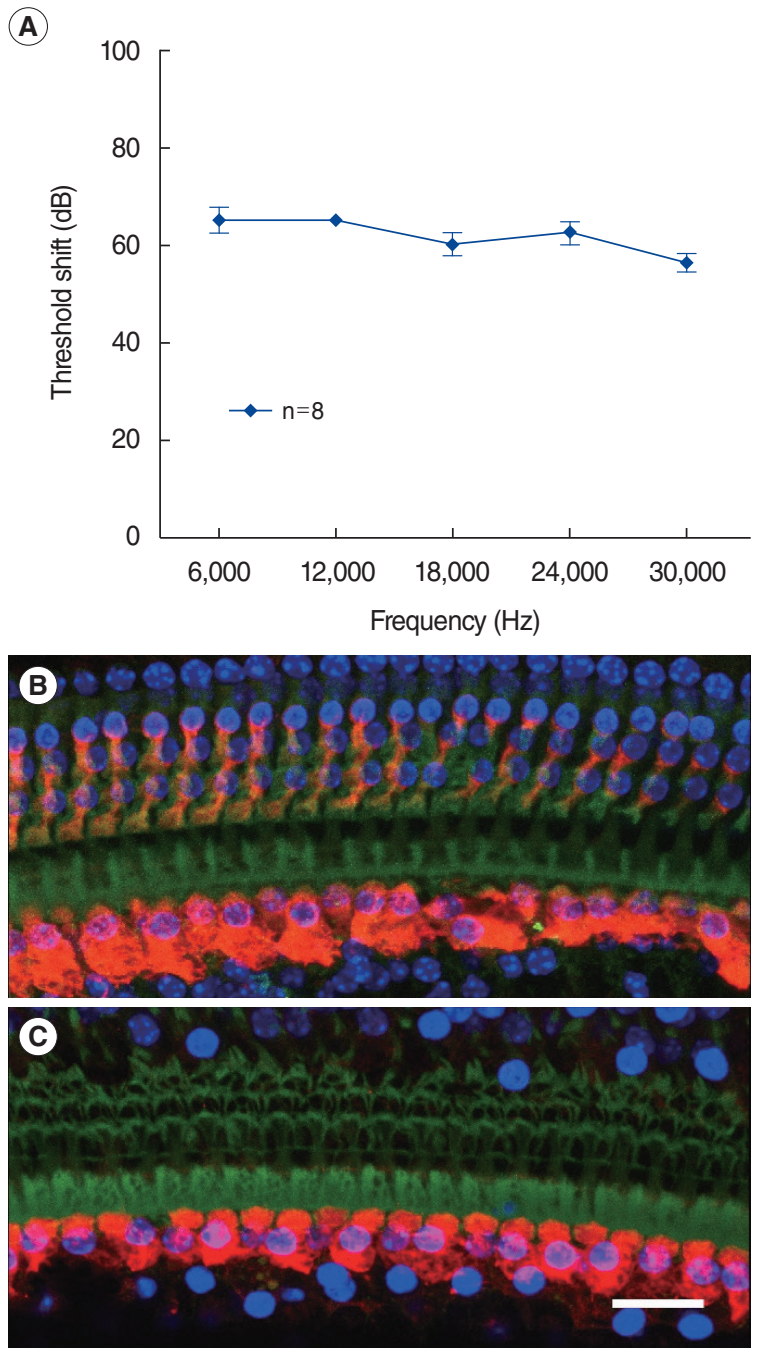

Fig. 1. Permanent threshold shift. Mice were exposed to $120-\mathrm{dB}$ white noise for 1 hour. The findings 2 weeks after noise exposure are shown. (A) Auditory brainstem response threshold shift. (B) The basal turn of the unexposed cochlea shows preserved inner and outer hair cells. (C) The noise-exposed cochlea shows degenerated outer hair cells and intact inner hair cells. The loss of nuclei (DAPI) and cell bodies (Myo7A) indicates degenerated outer hair cells. Only a cuticular plate is observed. Green, phalloidin; Blue, DAPI; Red, Myo7A. Scale bar $=20 \mu \mathrm{m}$.
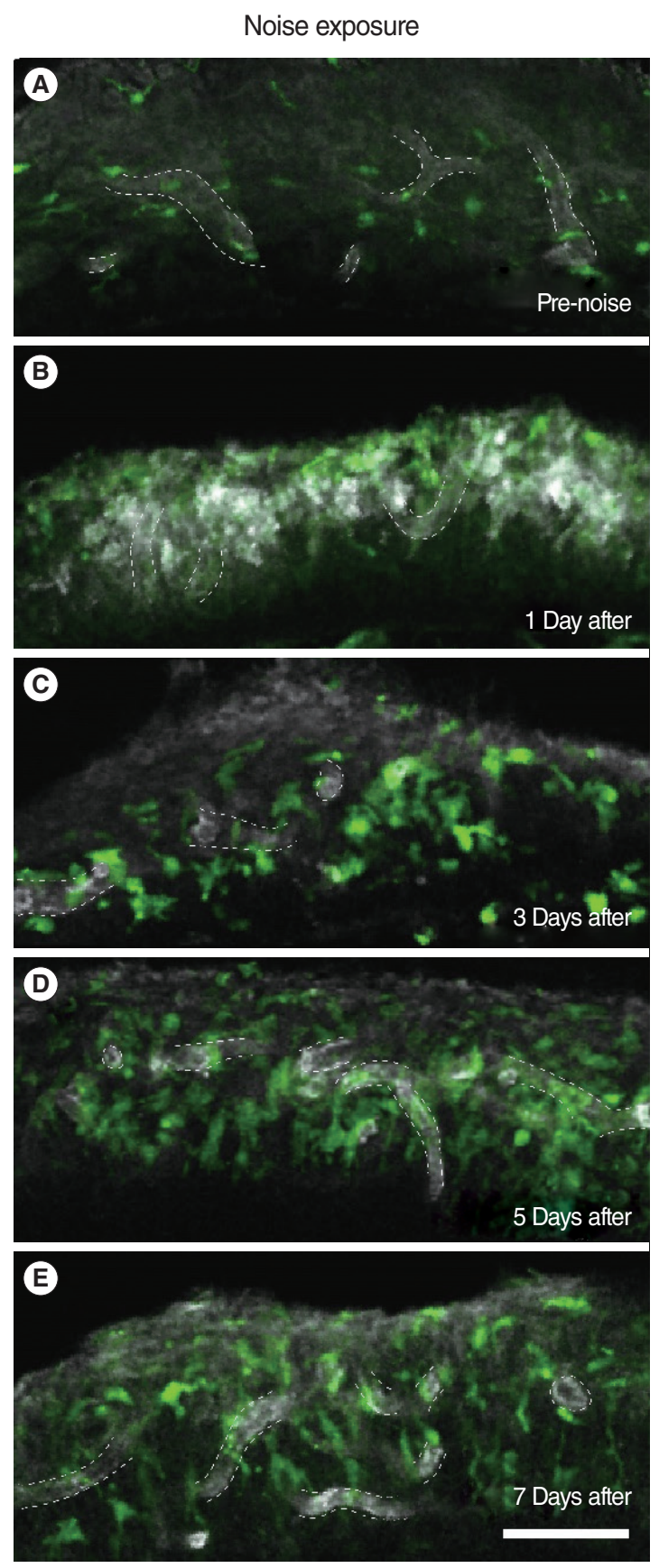

Fig. 2. Expression of monocyte markers in the basal turn of the cochlea. The lateral cochlear wall shows (A) CX3CR1+ resident macrophages before noise exposure and (B) a significant increase in Ly6Cpositive monocytes 1 day after noise exposure. (C) Three days after noise exposure, the cochlea displayed a decrease in Ly6C-positive monocytes and an increase in CX3CR $1^{+}$macrophages. The cochlea (D) 5, and (E) 7 days after noise exposure. The dim white color indicates non-specific staining of Ly6C to fibrocytes, and the weak white color with a hollow center indicates blood vessel endothelium, which is also known to express Ly6C. In all of the images, the top is the lateral wall and the bottom is the basilar membrane. Dotted line indicates Ly6C-stained endothelial cells of the blood vessel that are confirmed by exploration of z-stack images. Green, CX3CR1; White, Ly6C. Scale bar $=100 \mu \mathrm{m}$. 


\section{Statistical analysis}

For comparing multiple time points, a one-way analysis of variance test and post-hoc Tukey's test were used. For comparing two independent groups with multiple time points, a two-way analysis of variance test and post-hoc Dunnett's multiple comparison test were used. All graphs visualized the mean and standard error of the mean (error bar) values. IBM SPSS ver. 25.0 (IBM Corp., Armonk, NY, USA) and Prism 8.0 (GraphPad Software, San Diego, CA, USA) software were used for statistical analysis. A $P$-value $<0.05$ was regarded statistically significant.

\section{RESULTS}

\section{Intense noise causes an ABR threshold shift and outer hair} cell damage

We examined the ABR threshold shift and outer hair cell damage to study the effect of noise exposure on hair cells. Mice were continuously exposed to $120 \mathrm{~dB}$ peak equivalent SPL for 1 hour. Within 24 hours after noise exposure, the ABR thresholds of all frequencies were over $90 \mathrm{~dB}$. Two weeks after noise exposure, there was no improvement in hearing thresholds across all examined frequencies (Fig. 1A), indicating that the noise level generated a permanent threshold shift. Next, whole-mount cochlear preparations were made 2 weeks after the noise exposure. Hair cells were stained with the myosin-VIIa antibody and Alexa Fluor 488-phalloidin, and observed under a confocal microscope. In the unexposed cochleae, all outer hair cells were preserved (Fig. 1B), whereas most of them were damaged in the basal section of the noise-exposed cochlea (Fig. 1C).

\section{Infiltration of inflammatory monocytes}

We visualized the monocytes infiltrating the cochlea after noise exposure by Ly6C immunofluorescence. Ly6C is a membrane marker of inflammatory monocytes $[9,11,16,17]$. In the spiral ligament, Ly6C-positive monocytes were easily identified on day 1 after noise exposure compared to days 3,5 , and 7 or the unexposed cochleae (Fig. 2).

Next, we confirmed the monocyte infiltration after noise exposure by flow cytometry, because it is challenging to quantify the clustered infiltrated monocytes on immunofluorescence images. A serial analysis by gating for $\mathrm{CD} 11 \mathrm{~b}^{+}, \mathrm{Ly}_{6 \mathrm{G}}{ }^{-}, \mathrm{Ly}_{6} \mathrm{C}^{\text {high }}$ (indicators of inflammatory monocytes) showed a peak in the infiltration of inflammatory monocytes on day 1 post-exposure, followed by a subsequent decrease (Fig. 3) [17-21]. Leukocyte gating was performed with forward and side scatter signals, followed by gating for myeloid cells [22-24]. The CD11b antibody was used to gate the myeloid lineage immune cells, and the Ly6G
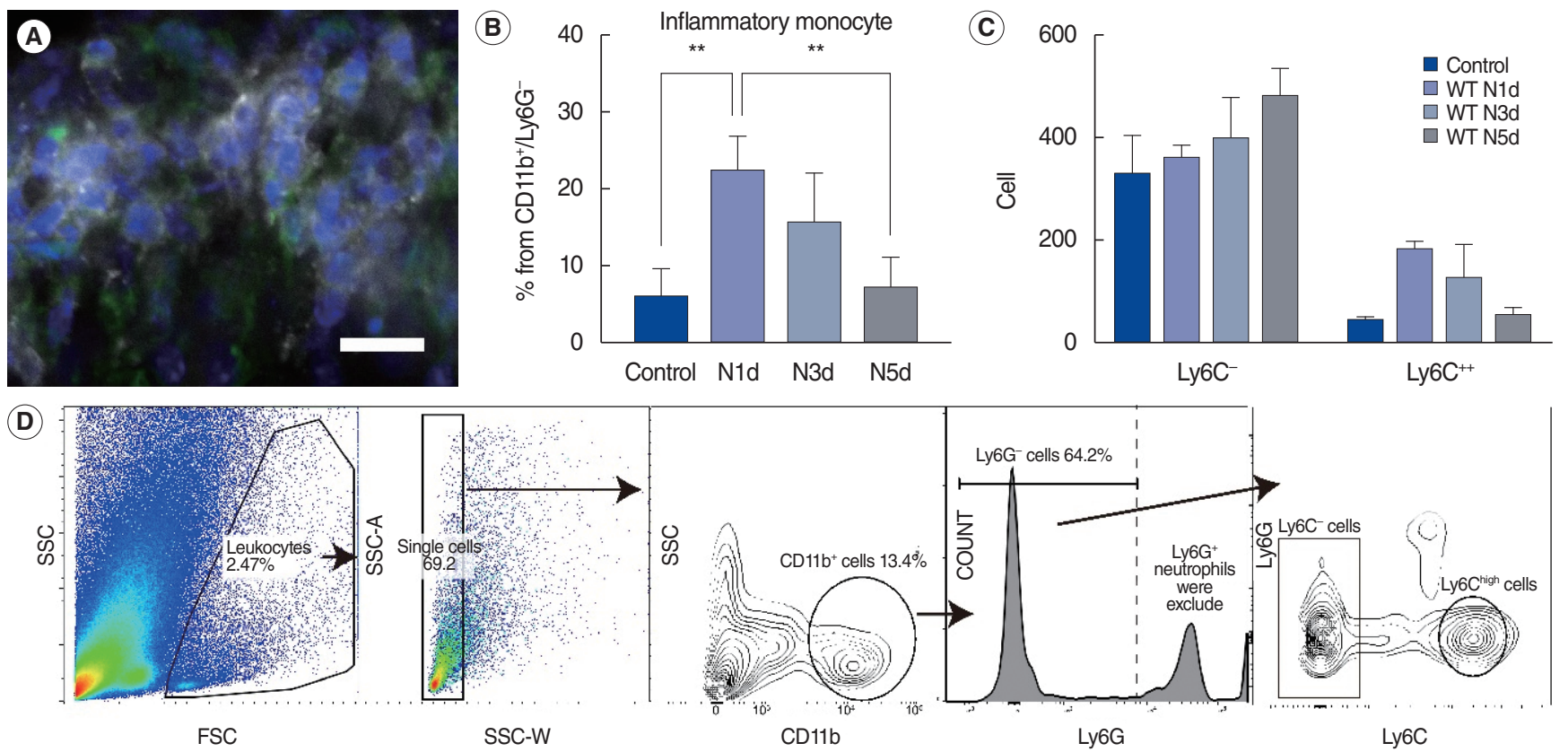

Fig. 3. Flow cytometric evaluation of the infiltrated monocytes after noise exposure. (A) Ly6C (white) and DAPI (blue) staining of the infiltrated monocytes in the spiral ligament after noise exposure indicates that Ly6C staining is not an artifact or background noise. Green, CX3CR1; White, Ly6C; and Blue, DAPI. Scale bar $=20 \mu \mathrm{m}$. (B) The percentage of monocytes (CD11 b+, Ly6G-, Ly6C high) from the parent population $\left(\mathrm{CD}_{11} \mathrm{~b}^{+}, \mathrm{Ly}_{6 \mathrm{G}}{ }^{-}\right){ }^{* \star} P<0.01$ by one-way analysis of variance with the Tukey post-hoc test). (C) The absolute cell count for $\mathrm{CD}^{*} 1 \mathrm{~b}^{+} / \mathrm{Ly} 6 \mathrm{G}^{-} / \mathrm{Ly} 6 \mathrm{C}^{-}$ cells and monocytes (CD11 b+ $\mathrm{b}_{6 \mathrm{G}}^{-}$, Ly6C high). (D) Gating for CD11b (myelocyte lineage) and Ly6G (neutrophils) to visualize monocyte infiltration. Ly6G+ cells were excluded because they were neutrophils. N1d, 1 day after noise exposure; N3d, 3 days after noise exposure; N5d, 5 days after noise exposure; WT, wild type; SSC, side scatter; FSC, forward scatter; SSC-A, side scatter area; SSC-W, side scatter width. 

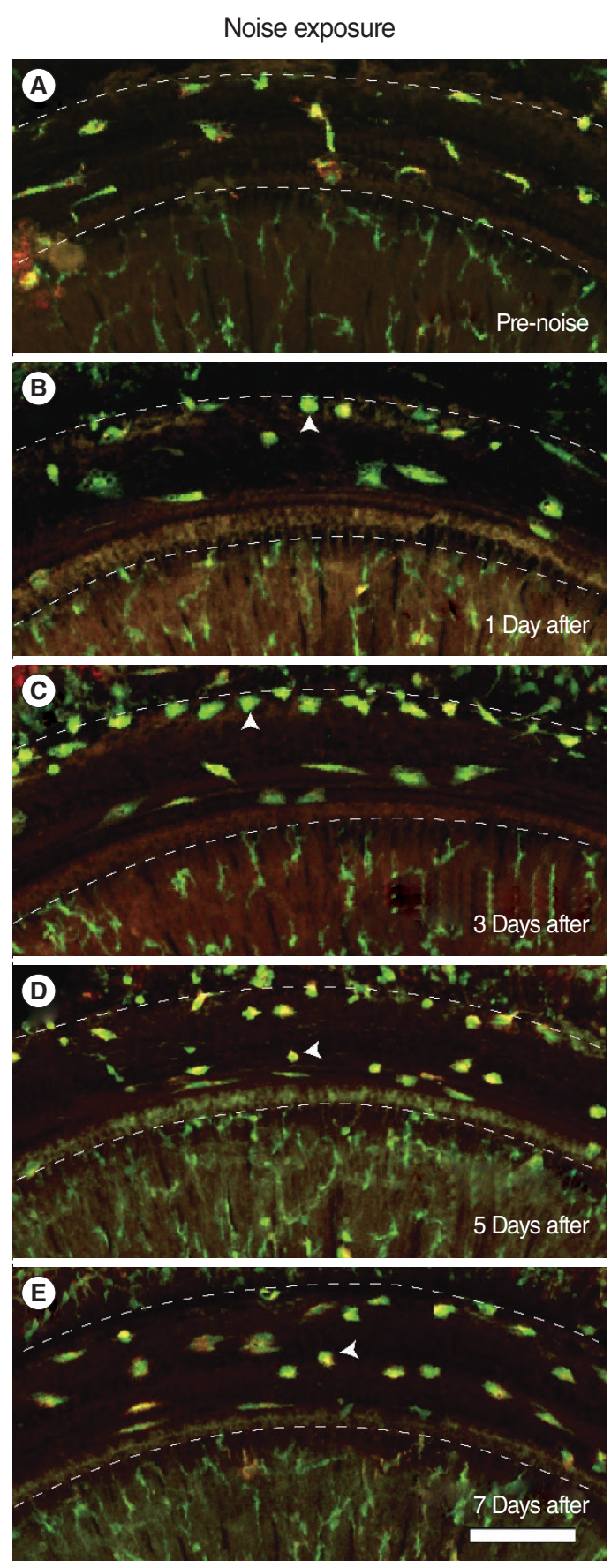

Fig. 4. Migration of macrophages after acoustic injury. Whole mounted images of the basilar membrane using a confocal microscope at each time point are presented. Several CX3CR1 ${ }^{+}$resident macrophages are seen beneath the basilar membrane in (A) unexposed cochlea and (B) 1 day after noise exposure. (C) Three days postnoise exposure, an increase in $\mathrm{CX} 3 \mathrm{CR} 1^{+}$cells with amoeboid morphology was seen in the junction of the basilar membrane and lateral wall (crista basilaris). Macrophages spread to the basilar membrane (D) 5, and (E) 7 days after noise exposure. All images show the lateral wall at the top and the osseous spiral lamina at the bottom. Arrowheads indicate representative amoeboid macrophages. Dotted lines indicate the borders of the basilar membrane. Green, CX3CR1; Red, F4/80; Scale bar $=100 \mu \mathrm{m}$.

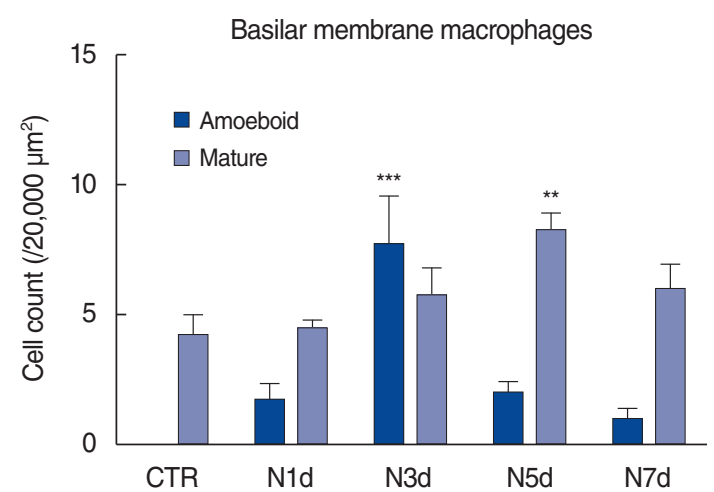

Fig. 5. Quantification of basilar membrane macrophages from four samples. Amoeboid macrophages were defined as dense, round cells without dendrites. Mature macrophages were defined as long, irregular shapes with dendrites. Cells in the basal turn (up to $60 \%$ of the distance from the apex) were counted. Two-way analysis of variance with the post-hoc Dunnett comparison was used to evaluate cell count differences from control cochlea. CTR, untreated cochlea; N1d, 1 day after noise exposure; N3d, 3 days after noise exposure; N5d, 5 days after noise exposure; N7d, 7 days after noise exposure. ${ }^{\star \star} P<0.01,{ }^{\star \star *} P<0.001$.

antibody was used to exclude neutrophils because they also weakly express Ly6C [21]. The other cells in the $\mathrm{CD}^{11} \mathrm{~b}^{+} / \mathrm{Ly} 6 \mathrm{G}^{-} / \mathrm{Ly} 6 \mathrm{C}^{-}$ population consisted of dendritic cells, eosinophils, natural killer cells, and mostly macrophages [25]. Although there was no statistical significance, the absolute cell count of the $\mathrm{CD} 11 \mathrm{~b}^{+} / \mathrm{Ly}^{6} \mathrm{G}^{-} /$ Ly6 $\mathrm{C}^{-}$population gradually increased after noise, which may have resulted from the transformation of monocytes to macrophages, based on evidence from previous studies [22,25].

\section{Macrophages migrate to the basilar membrane from the lateral wall}

Based on whole-mount immunofluorescence performed on consecutive days after noise exposure, we investigated the migration of macrophages to the basilar membrane (Fig. 4, Supplementary Fig. 1). All the CX3CR1-positive (green) macrophages were costained with F4/80 antibody (red) throughout the entire field of vision. On day 1 after noise exposure, the cochlea showed no remarkable changes in the number and morphology of CX3CR1positive macrophages displaying long and irregular shapes. However, on day 3 after noise exposure, the number of CX3CR1positive macrophages increased, and they were lined along the crista basilaris (junction of the basilar membrane and lateral wall). These cells displayed round and amoeboid morphology, suggesting that they were newly infiltrated monocytes [4,5]. On days 5 and 7 after noise exposure, the CX3CR1-positive macrophages did not line the crista basilaris; instead, they had spread to the basilar membrane. The predominant morphology of macrophages at these time points was not amoeboid, but rather dendritic and irregular-shaped (Fig. 5). 


\section{DISCUSSION}

Our findings revealed that the inflammatory monocytes migrated to the spiral ligament 1 day after noise exposure. In contrast, a change in the number of macrophages could be identified from day 3 onwards after noise exposure, based on immunofluorescence. The time taken for this population change suggests that the infiltrated monocytes transformed into macrophages. The crista basilaris is likely the major site of transformation since amoeboid macrophages lined this area 3 days after noise exposure. On days 5 and 7, the macrophages spread to the basilar membrane, potentially eliminating the degenerated cell debris and healing the damaged tissue.

Resident macrophages are present in the spiral ligament, spiral limbus, and spiral ganglion region, as well as beneath the basilar membrane, displaying various morphologies in different regions of the cochlea $[3,6,26,27]$. Previous studies have shown that while resident macrophages do not proliferate, circulating monocytes enter the cochlea and differentiate into mature macrophages after noise exposure. Hirose et al. [2] argued against macrophage proliferation in the spiral ligament based on immunohistochemical analyses after BrdU injections into mice following noise exposure. Okano et al. [6] also showed that transplanting GFP-labeled bone marrow into lethally irradiated mice resulted in an increased number of $\mathrm{GFP}^{+}$macrophages in the cochlea. Consistent with these findings, we demonstrated that the cochlear macrophage population was likely increased by the migration of monocytes, which are macrophage precursors, from the vasculature- - not by mitotic division of the resident macrophages.

Previous studies have shown that $\mathrm{CX} 3 \mathrm{CR} 1^{+}$and $\mathrm{CD} 45^{+}$cells in the cochlea peak at 3 days post-noise exposure [2,6,7]. Yang et al. [4] showed an accumulation of cells displaying amoeboid morphology in the junction between the basilar membrane and lateral wall 4 days post-noise exposure. In line with these findings, we found several CX3CR1 ${ }^{+}$cells with amoeboid morphology in the crista basilaris 3 days after noise exposure, whereas they were not seen in the unexposed cochlea or 1 day after noise exposure. Instead, we found inflammatory monocytes 1 day after noise exposure. To the best of our knowledge, this is the earliest observation of immune cell infiltration after noise exposure. Our novel observations can explain the time course of the increase in the macrophage population after acoustic trauma, which has not previously been characterized. Monocytes from the blood started to infiltrate the cochlea parenchyma at 1 day after noise, and gradually decreased. They then began to transform into macrophages while losing the Ly6C phenotype at 3 days after noise exposure, which is consistent with the findings observed by other researchers, as described above.

Ly6C has been recently identified as an important marker of monocytes. Ly6 $\mathrm{C}^{\text {high }}$ monocytes display an inflammatory phenotype, while Ly $6 \mathrm{C}^{\text {low }}$ monocytes are categorized as patrolling monocytes in the bloodstream $[9,11,16,17]$. We defined Ly6 $\mathrm{C}^{\text {high }}$ $\left(\mathrm{CD}_{11 b^{+}}\right.$, Ly6G $\left.\mathrm{G}^{-}, \mathrm{Ly}_{6} \mathrm{C}^{\text {high }}\right)$ cells as infiltrating monocytes based on previous studies [17-21]. In our study, Ly6 $\mathrm{C}^{\text {high }}$ monocytes accumulated in the lateral wall from the first day after noise exposure. These infiltrating monocytes transform into macrophages or dendritic cells in other organs $[17,19,20]$. Our findings, therefore, indicate that the cochlea has characteristics similar to other organs, allowing the transformation of monocytes into macrophages.

This study has some limitations. First, direct evidence regarding the transformation of monocytes into macrophages is still lacking. Therefore, future studies should track monocytes and gather meticulous FACS data. In the FACS analysis, the proportion of leukocytes in the total cell population was very low. The results may have been biased by contamination with non-leukocyte cells. Enrichment processes or additional cellular markers may be applied for more accurate analyses in future studies. Second, we focused on inflammatory monocytes, which are widely known to be involved in cochlear inflammation. However, other resident immune cells have also been reported in the cochlea after noise exposure [28]. The interaction of these cells with the monocytes that infiltrate following noise exposure needs to be evaluated.

In conclusion, we have demonstrated that acoustic overstimulation leads to the migration of circulating inflammatory monocytes into the lateral cochlear wall, which peaks 1 day after noise exposure. These infiltrated inflammatory monocytes acquire the properties of macrophages near the crista basilaris before spreading to the basilar membrane.

\section{CONFLICT OF INTEREST}

No potential conflict of interest relevant to this article was reported.

\section{ACKNOWLEDGMENTS}

This research was supported by the Basic Science Research Program through the National Research Foundation of Korea (NRF) and funded by the Ministry of Education (NRF-2020R1I1A1A01067241 to SHB).

\section{ORCID}

Seung Ho Shin

Jee Eun Yoo

Jinsei Jung

Jae Young Choi

Seong Hoon Bae https://orcid.org/0000-0001-9352-8426 https://orcid.org/0000-0001-9372-999X https://orcid.org/0000-0003-1906-6969 https://orcid.org/0000-0001-9493-3458

https://orcid.org/0000-0001-9243-9392 


\section{AUTHOR CONTRIBUTIONS}

Conceptualization: SHB. Data curation: SHB, SHS, JEY. Formal analysis: SHS. Funding acquisition: SHB. Methodology: SHB, SHS, JEY. Project administration: SHB. Visualization: SHS. Writing-original draft: SHB, SHS. Writing-review \& editing: JYC, JJ.

\section{SUPPLEMENTARY MATERIALS}

Supplementary materials can be found via https://doi.org/10. 21053/ceo.2021.00857.

\section{REFERENCES}

1. Wang Y, Hirose K, Liberman MC. Dynamics of noise-induced cellular injury and repair in the mouse cochlea. J Assoc Res Otolaryngol. 2002 Sep;3(3):248-68.

2. Hirose K, Discolo CM, Keasler JR, Ransohoff R. Mononuclear phagocytes migrate into the murine cochlea after acoustic trauma. J Comp Neurol. 2005 Aug;489(2):180-94.

3. Lang H, Ebihara Y, Schmiedt RA, Minamiguchi H, Zhou D, Smythe $\mathrm{N}$, et al. Contribution of bone marrow hematopoietic stem cells to adult mouse inner ear: mesenchymal cells and fibrocytes. J Comp Neurol. 2006 May;496(2):187-201.

4. Yang W,Vethanayagam RR, Dong Y, Cai Q, Hu BH.Activation of the antigen presentation function of mononuclear phagocyte populations associated with the basilar membrane of the cochlea after acoustic overstimulation. Neuroscience. 2015 Sep;303:1-15.

5. He W, Yu J, Sun Y, Kong W. Macrophages in noise-exposed cochlea: changes, regulation and the potential role. Aging Dis. 2020 Feb;11(1): 191-9.

6. OkanoT, NakagawaT, KitaT, Kada S, Yoshimoto M, NakahataT, et al. Bone marrow-derived cells expressing Iba1 are constitutively present as resident tissue macrophages in the mouse cochlea. J Neurosci Res. 2008 Jun;86(8):1758-67.

7. Wood MB, Zuo J.The contribution of immune infiltrates to ototoxicity and cochlear hair cell loss. Front Cell Neurosci. 2017 Apr;11:106.

8. Hu BH, Zhang C, Frye MD. Immune cells and non-immune cells with immune function in mammalian cochleae. Hear Res. 2018 May;362: 14-24.

9. Auffray C, Sieweke MH, Geissmann F. Blood monocytes: development, heterogeneity, and relationship with dendritic cells. Annu Rev Immunol. 2009;27:669-92.

10. Gallagher KA, Joshi A, CarsonWF, Schaller M, Allen R, Mukerjee S, et al. Epigenetic changes in bone marrow progenitor cells influence the inflammatory phenotype and alter wound healing in type 2 diabetes. Diabetes. 2015 Apr;64(4):1420-30.

11. Willenborg S, Lucas T, van Loo G, Knipper JA, Krieg T, Haase I, et al. CCR2 recruits an inflammatory macrophage subpopulation critical for angiogenesis in tissue repair. Blood. 2012 Jul;120(3):613-25.

12. Wynn TA, Vannella KM. Macrophages in tissue repair, regeneration, and fibrosis. Immunity. 2016 Mar;44(3):450-62.
13. Kaur T, Zamani D, Tong L, Rubel EW, Ohlemiller KK, Hirose K, et al. Fractalkine signaling regulates macrophage recruitment into the cochlea and promotes the survival of spiral ganglion neurons after selective hair cell lesion. J Neurosci. 2015 Nov;35(45):15050-61.

14. Tan WJ, Thorne PR, Vlajkovic SM. Characterisation of cochlear inflammation in mice following acute and chronic noise exposure. Histochem Cell Biol. 2016 Aug;146(2):219-30.

15. Bae SH, Kwak SH,Yoo JE, Kim KM, Hyun YM, Choi JY, et al.Threedimensional distribution of cochlear macrophages in the lateral wall of cleared cochlea. Clin Exp Otorhinolaryngol. 2021 May;14(2): 179-84.

16. Italiani P, Boraschi D. From monocytes to M1/M2 macrophages: phenotypical vs. functional differentiation. Front Immunol. 2014 Oct; 5:514.

17. Nahrendorf M, Swirski FK, Aikawa E, Stangenberg L, Wurdinger T, Figueiredo JL, et al. The healing myocardium sequentially mobilizes two monocyte subsets with divergent and complementary functions. J Exp Med. 2007 Nov;204(12):3037-47.

18. Dunay IR, Damatta RA, Fux B, Presti R, Greco S, Colonna M, et al. Gr1(+) inflammatory monocytes are required for mucosal resistance to the pathogen Toxoplasma gondii. Immunity. 2008 Aug;29(2): 306-17.

19. Kimball AS, Joshi A, Carson WF 4th, Boniakowski AE, Schaller M, Allen R, et al. The histone methyltransferase MLL1 directs macrophage-mediated inflammation in wound healing and is altered in a murine model of obesity and type 2 diabetes. Diabetes. 2017 Sep; 66(9):2459-71.

20. Nahrendorf M, Pittet MJ, Swirski FK. Monocytes: protagonists of infarct inflammation and repair after myocardial infarction. Circulation. 2010 Jun;121(22):2437-45.

21. Rose S, Misharin A, Perlman H. A novel Ly6C/Ly6G-based strategy to analyze the mouse splenic myeloid compartment. Cytometry A. 2012 Apr;81(4):343-50.

22. Kimball A, Schaller M, Joshi A, Davis FM, denDekker A, Boniakowski A, et al. Ly6CHi blood monocyte/macrophage drive chronic inflammation and impair wound healing in diabetes mellitus. ArteriosclerThromb Vasc Biol. 2018 May;38(5):1102-14.

23. Ghasemlou N, Chiu IM, Julien JP,Woolf CJ. CD11b+Ly6G- myeloid cells mediate mechanical inflammatory pain hypersensitivity. Proc Natl Acad Sci U S A. 2015 Dec;112(49):E6808-17.

24. Loken MR, Brosnan JM, Bach BA, Ault KA. Establishing optimal lymphocyte gates for immunophenotyping by flow cytometry. Cytometry. 1990;11(4):453-9.

25. Liyanage SE, Gardner PJ, Ribeiro J, Cristante E, Sampson RD, Luhmann UF, et al. Flow cytometric analysis of inflammatory and resident myeloid populations in mouse ocular inflammatory models. Exp Eye Res. 2016 Oct;151:160-70.

26. Sato E, Shick HE, Ransohoff RM, Hirose K. Repopulation of cochlear macrophages in murine hematopoietic progenitor cell chimeras: the role of CX3CR1. J Comp Neurol. 2008 Feb;506(6):930-42.

27. Shi X. Resident macrophages in the cochlear blood-labyrinth barrier and their renewal via migration of bone-marrow-derived cells. Cell Tissue Res. 2010 Oct;342(1):21-30.

28. Rai V, Wood MB, Feng H, Schabla NM, Tu S, Zuo J. The immune response after noise damage in the cochlea is characterized by a heterogeneous mix of adaptive and innate immune cells. Sci Rep. 2020 Sep;10(1):15167. 